\title{
Common-mode Backchannel Signaling System for Differential High-speed Links
}

\author{
Andrew Ho ${ }^{1}$, Vladimir Stojanović ${ }^{1,2}$, Fred Chen ${ }^{1}$, Carl Werner ${ }^{1}$, Grace Tsang ${ }^{1}$, Elad Alon ${ }^{1,2}$, \\ Ravi Kollipara ${ }^{1}$, Jared Zerbe ${ }^{1}$, and Mark A. Horowitz ${ }^{2}$ \\ ${ }^{\mathrm{I}}$ Rambus, Inc., Los Altos, CA 94022, USA \\ ${ }^{2}$ Department of Electrical Engineering, Stanford University, CA 94305, USA
}

\begin{abstract}
Common-mode signaling is effectively used to create a backchannel communication path over the existing pair of wires for a self-contained adaptive differential high-speed link transceiver cell [1]. A transceiver chip was designed in $0.13 \mu \mathrm{m}$ CMOS to demonstrate the feasibility of simultaneous differential and common-mode signaling. The design uses a three-level return-to-null signaling scheme with simultaneous voltage and timing reference extraction, to minimize the hardware costs and achieve robust operation for sending update information from receiver to the transmitter. The measured results indicate that this backchannel achieves reliable communication without noticeable impact on the forward link for bandwidths up to $50 \mathrm{MHz}$ and swings of 20-100mV.
\end{abstract}

Keywords: Common-mode, backchannel, high-speed links.

\section{Introduction}

With signaling rates in high-speed links on the rise, communication techniques such as equalization and multilevel modulation are increasingly used to mitigate the effects of band-limited channels. However, integration of these techniques greatly complicates link configuration. While transmit pre-emphasis is often used to implement equalization in high-speed links, adaptively configuring the transmit filter for an a priori unknown channel requires communication from the corresponding receiver $[1,2]$. A backchannel allows for this communication path from the receiver to the transmitter whereby updates can be made to transmit equalization coefficients based on received data.

Existing backchannel techniques impose requirements at the system level, such as specific link arrangements [2] or the data routing capability of centralized control. This paper explores integrating the backchannel as part of the link transceiver to create a self-contained cell, avoiding constraints on the system by exploiting the common-mode signaling dimension [3] of the forward differential channel.

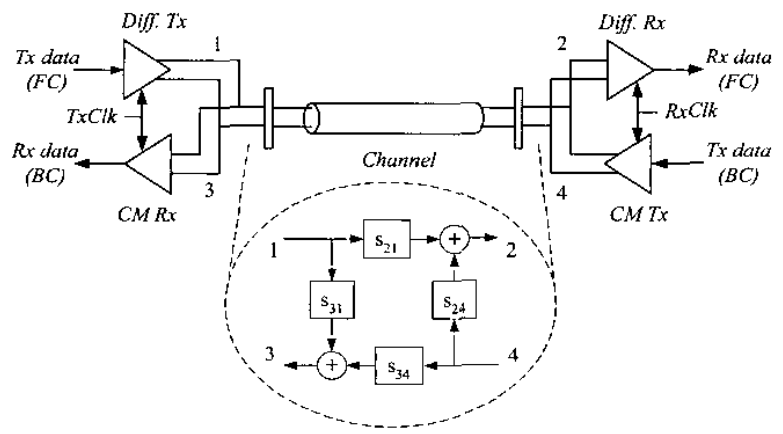

Fig. 1 Simultaneous common-mode and differential signaling system block diagram.
We show that because of the signal integrity issues arising from simultaneous differential and common-mode signaling, the backchannel must use limited swing and tolerate relatively large noise sources. Coupled with issues from the inherent single-ended nature of common-mode signals, these constraints drive the design of an edge-based signaling system with simultaneous timing and voltage reference extraction. We implemented this system as part of a $0.13 \mu \mathrm{m}$ high-speed link transceiver, based on our previous design [4], to address the signaling issues and verify the backchannel system design.

\section{Simultaneous Common-mode and Differential Signaling}

The system block diagram with differential forward channel (FC) and common-mode backchannel (BC) is shown in Fig. 1. In theory, the differential and common-mode signals are orthogonal. In practice, inevitable non-idealities lead to signal coupling between modes, causing signal integrity degradation in both domains. The transfer functions $s_{21}$ and $\mathrm{S}_{34}$ are the channel response of the differential forward channel and common-mode backchannel, respectively. The crosstalk of the differential transmitter to the common-mode receiver is represented by the $s_{31}$ transfer function. The coupling of the backchannel transmitter to the forward channel receiver corresponds to the $s_{24}$ transfer function.

\section{A. Effect of common-mode signal on differential signal}

Differential signals generated by uncorrelated commonmode transmission appear as noise to the differential receiver, degrading the signal integrity of the forward channel. This common-mode-to-differential conversion is, in part, a result of mismatch in the passive components (connectors, vias, backplane traces, etc.) and can be simply analyzed as crosstalk. Measured $\mathrm{s}_{24}$ of a typical 26" FR4 backplane bottom trace and linecards with two connectors, Fig. 2, shows the significant increase in this crosstalk beyond $100 \mathrm{MHz}$.

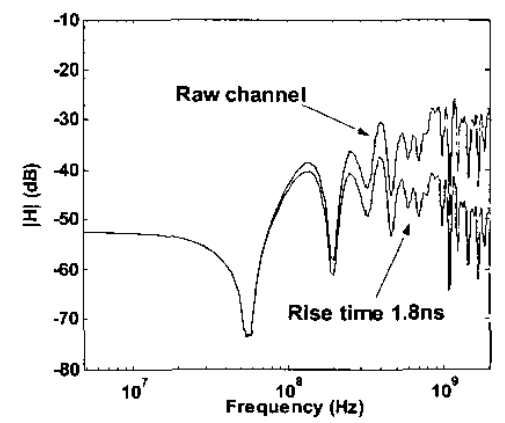

Fig. 2 Measured common-mode transmitter to differential receiver crosstalk transfer function $\left(\mathrm{s}_{24}\right)$ of a typical 26" FR4 backplane bottom trace and linecards with HSD connectors, with and without increased rise time in the backchannel transmitter. 
To minimize the impact of this crosstalk on the forward link, we increased the rise time of the common-mode transmitter. Increasing rise time for the same swing magnitude has a similar effect to adding a low-pass filter to the transmitter output with a lower cutoff frequency. The crosstalk response with the implemented increased rise time is overlaid in Fig. 2. Slower rise time attenuates crosstalk due to mismatch in passive components for frequencies outside of the backchannel bandwidth. Since crosstalk is a proportional noise source, the backchannel swing can be decreased to further mitigate the crosstalk injected onto the forward channel.

Another cause of forward link margin degradation is the effect of the common-mode signal on the differential receiver's offset and sensitivity. Fig. 3(a) shows the measured offset over a range of common-mode voltages for the differential receiver. Static offset can be removed with onetime digital offset cancellation. Dynamic changes in offset directly reduce the voltage margin of the differential receiver, unless the receiver includes dynamic offset cancellation. Fig. 3 (b) presents measured differential receiver sensitivity as a function of receiver common-mode voltage. Reduction in receiver sensitivity due to common-mode variations will also decrease the voltage margin of the forward link. To mitigate this degradation, the common-mode signal magnitude and range should be chosen so that the corresponding range of offset and sensitivity change is within the noise budget. With a fixed forward link noise budget, backchannel signal magnitude is maximized by choosing the operating point to be around the plateaus of Fig. 3(a) and 3(b).

To satisfy the tight noise budget in high performance applications, the backchannel signal is limited to a very small swing relative to the forward signal. Our design has a programmable peak-to-peak common-mode signal swing of $12-100 \mathrm{mV}$ to allow tradeoffs between forward-channel and backchannel reliability.

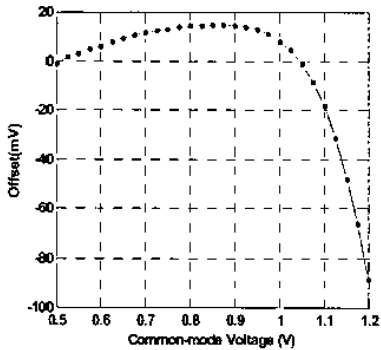

(a)

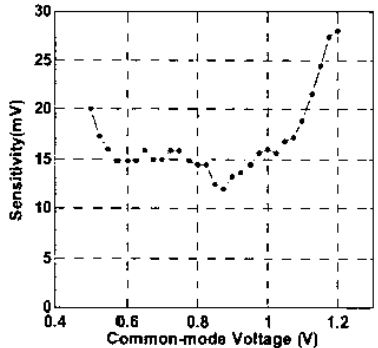

(b)
Fig. 3 (a) Measured differential receiver offset versus common-mode voltage, (b) Measured differential receiver sensitivity versus common-mode voltage.

\section{B. Effect of differential signal on common-mode signal}

Differential-to-common-mode conversion can severely degrade the signal integrity of the low-swing backchannel. This conversion, in part, is a result of mismatch in the passive components of the link. The $s_{31}$ crosstalk transfer function is shown in Fig. 4. A band-limited preamp in the backchannel receiver acts as a two-pole low-pass filter to reduce the impact of this crosstalk.

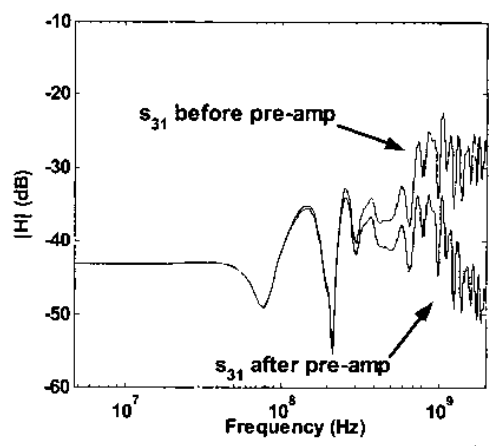

Fig. 4 Measured crosstalk of differential transmitter to commonmode receiver $\left(\mathrm{s}_{31}\right)$ of a typical 26 " FR4 backplane bottom trace and linecards with HSD connectors, before and after a two-pole low-pass preamp filter at $650 \mathrm{MHz}$ in the backchannel receiver.

In addition to the crosstalk caused by mode conversion, the differential transmitter generates common-mode noise. Fig. 5 shows the measured frequency response of the common-mode glitch caused by the asymmetrical rising and falling edges of each leg in a differential transmitter. The glitch happens at every forward channel bit transition, with most of its energy concentrated in the frequency range outside that of the backchannel bandwidth. Like $s_{31}$, the frequency content of the glitch that is in the band of the common-mode receiver directly interferes with the backchannel. The low-pass filter added to reduce crosstalk also reduces the amount of differential transmitter inflicted common-mode glitch noise seen by the backchannel receiver.

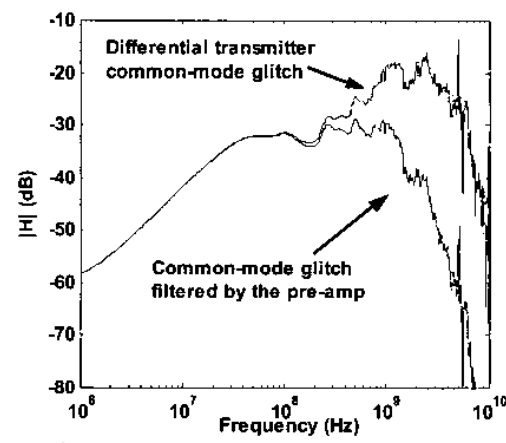

Fig. 5 Measured frequency responses of the common-mode glitch generated by the differential transmitter before and after a two-pole low-pass preamp filter at $650 \mathrm{MHz}$ are shown.

As the backchannel signal magnitude is restricted to minimize interference with the forward link and to contain electro magnetic interference, the total common-mode noise, even after the low-pass filter, can be on the same order as the backchannel swing. Since the backchannel is only used to adapt the transmitter's configuration, error rates as large as $30 \%$ can be tolerated (as shown later in the result section).

\section{Signaling Scheme}

Our receiver must extract both a timing reference and voltage reference from the incoming signal in a high-noise environment. Timing synchronization is complicated by the fact that the two chips may not share a common reference clock. Voltage reference extraction is complicated by the possibility of AC-coupled or DC-coupled link operation.

Fortunately, the required backchannel bandwidth is considerably lower than that of the forward channel since the 
backchannel is only used to send packets of update information for the differential transmit equalizer coefficients. The relatively low backchannel frequency allows the use of a simple return-to-null signaling scheme and an oversampling receiver for timing reference extraction. Referring to Fig. 6, backchannel data is encoded on a tri-level signal with the null as the center level. Since each bit has two transitions, timing recovery is simplified.

Simple edge detection, achieved by differentiating the oversampled digital output, provides both data extraction and synchronization. The oversampling rate is great enough to provide high tolerance to frequency offset variations. In our design, we used an over-sampling ratio of $20 \mathrm{x}$. The differentiator is implemented with a voltage tracking loop at the receiver (Fig. 8); the voltage reference is extracted by using the oversampled digital output as feedback to track the common-mode signal via an integrator. Edges are detected when the number of 1's (for a rising edge) or 0's (for a falling edge) from the oversampled common-mode signal in a fixed size window exceeds a configurable threshold. As an additional benefit, offset in the common-mode receiver and slow variations in common-mode (e.g. baseline wander in AC-coupled systems) are cancelled in the process of signal tracking.

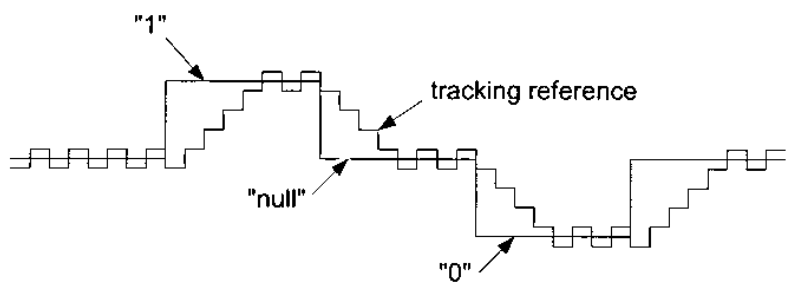

Fig. 6 Oversampled, edge-encoded 3-level signaling with tracking receiver (three characters are denoted as a " 1 ", " 0 " and "null").

\section{Implementation}

To minimize impact on the forward differential link, a common-mode transmitter must provide both high-output impedance and low parasitic capacitance.

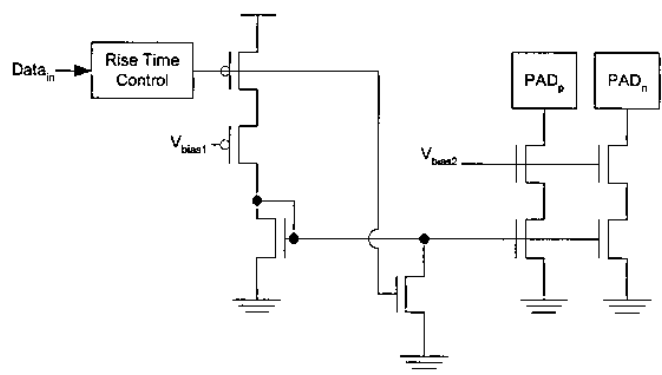

Fig. 7 Cascoded common-mode transmitter to minimize channel length modulation and common-mode-to-differential crosstalk.

As shown in Fig. 7, a pair of carefully matched cascode structures is used to reduce channel length modulation so that the difference in current drawn from PADp and PADn is as small as possible in the presence of the received differential signal. The relatively small cascode devices isolate the large parasitic capacitance of the bottom current source devices from the pads. Rise time control is implemented directly from the digital data signal using an undersized driver.
In the backchannel receiver, Fig. 8, a resistive divider connected to both inputs generates the common-mode voltage. To prevent degradation of the differential receiver termination, a pair of high-valued, carefully matched on-chip resistors is used. A two-stage differential preamplifier with a gain of 3 acts as a two-pole low-pass filter at $650 \mathrm{MHz}$ to reduce high frequency noise from the differential transmitter.

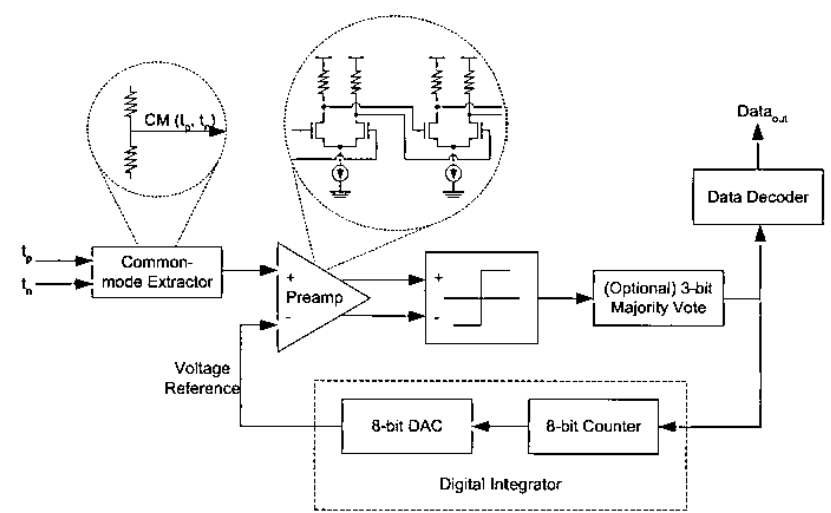

Fig. 8 Common-mode receiver block diagram. Two-stage preamp amplifies the common-mode signal and low-pass filters the high-frequency common-mode noise. Digital integrator provides both reference level extraction and offset cancellation.

To provide flexible backchannel bandwidth, the tracking receiver employs a digital integrator. The integrator consists of an 8-bit up-down counter and a digital-to-analog converter (DAC). The digital integrator provides good visibility and testability. It enables flexible backchannel data rate: as long as the DAC settling time does not extend beyond the sampling period, the tracking rate is solely determined by the clock that drives the integrator and the step size of the DAC.

The digital integrator requires a wide-range, highresolution, linear DAC to accurately track the low-swing common-mode signal. The wide range (up to $250 \mathrm{mV}$ ) is required for compatibility with AC-coupled and DC-coupled systems.

In this work, a pre-existing DAC circuit was re-used for the backchannel receiver. This DAC is the performance limiter in the backchannel system because of its large differential nonlinearity (DNL). This DNL results in an irrecoverable loss of voltage margin for the backchannel. The use of one coarse DAC to track the natural common-mode level set by the differential transmitter, and a second fine DAC to track the common-mode signal could relax the design constraints of a single wide-range, high resolution DAC.

Since the backchannel is a noisy signaling environment, a parity-check bit is added to each transmitted packet. This additional bit allows the detection of single-edge slips, which are common in edge-based systems.

\section{Experimental Results}

Fig. 9 shows the common-mode backchannel transmitted waveform and the relative tracking rate of the digital tracking loop. The digital loop tracks the transmitted backchannel pulses in approximately ten clock counts. The dithering at each plateau shows the bit boundaries as seen by the backchannel receiver. 


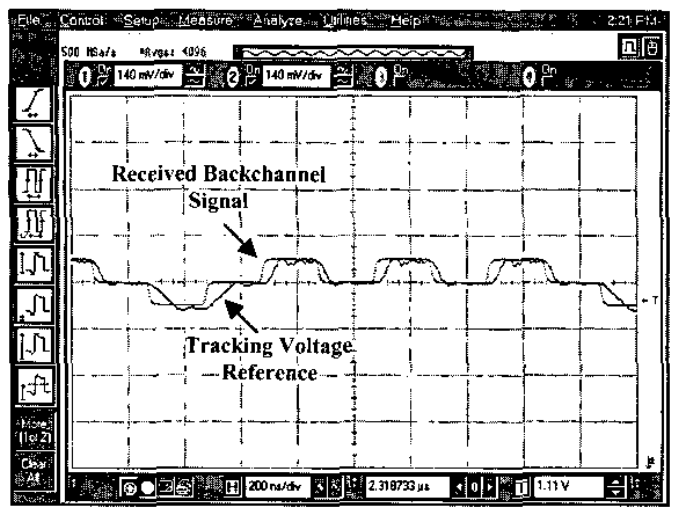

Fig. 9 Scope captured common-mode backchannel transmitted waveform overlaid with signal tracking of common-mode backchannel receiver.

The common-mode backchannel swing can be adjusted to provide a tradeoff between forward channel signal integrity and backchannel noise immunity. Fig. 10 shows the packet error and drop rates of the backchannel at different peak-topeak common-mode swings. A packet is dropped when an error in synchronization is detected (i.e. when the time between any two consecutive received bits in a packet exceeds a set threshold)

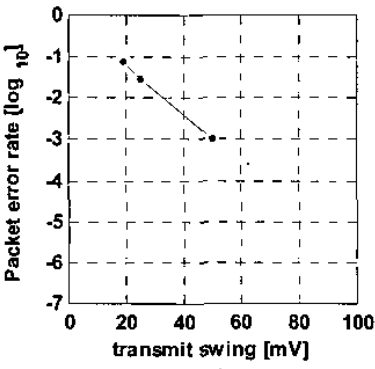

(a)

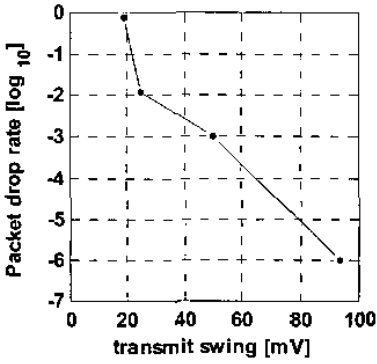

(b)
Fig. 10 (a) Packet error rate and (b) Packet drop rate as a function of peak-to-peak common-mode swing.

While packet error and drop rates are important for reliable transmission of various configuration and link information data, it is interesting to note that theoretically for adaptive algorithm convergence it is only necessary to have an error rate of less than $50 \%$. The packet drop rate only influences the total adaptation time. For example, Fig. 10 shows that packet drop rate decreases nearly two orders of magnitude when backchannel swing is increased from $18.75 \mathrm{mV}$ to $25 \mathrm{mV}$, so adaptation time improves rapidly. To illustrate this effect, we plot the convergence of transmit pre-emphasis taps as a function of the number of sent updates for these two backchannel swing amplitudes in Fig. 11. These plots indicate that effective adaptation is still possible even at very low backchannel amplitude. As indicated in Fig. 10, very reliable communication can be achieved for backchannel swings of $50 \mathrm{mV}$ and higher.

Even at the largest available backchannel swing of $100 \mathrm{mV}$, there was no measurable impact on the noise margin of the forward channel at a target BER of $10^{-12}$. This indicates that the noise induced by the backchannel on the forward channel is less than $2 \mathrm{mV}$ - the minimum change in margin detectable by our measurement setup.
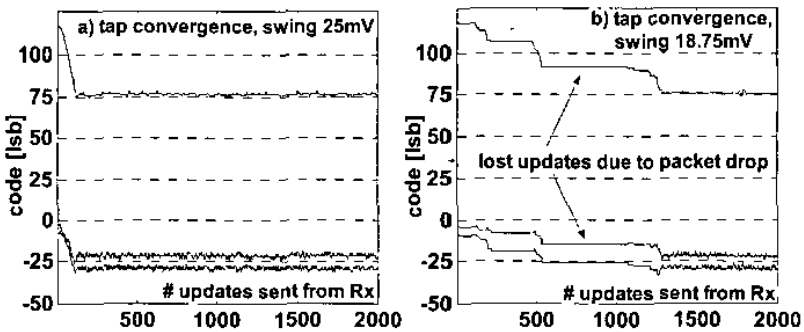

Fig. 11 Transmit pre-emphasis tap convergence: a) backchannel swing of $25 \mathrm{mV}$ with packet drop rate of $1 \%$ and packet error rate less than $5 \%$ b) backchannel swing of $18.75 \mathrm{mV}$ with packet error rate roughly $10 \%$ and packet drop rate of $80 \%$.

\section{Conclusion}

Common-mode signaling has been used for reverse direction data flow in a high-speed differential link application. Designing a backchannel solution that has low impact on the forward link, in terms of both complexity and signal integrity, requires the synergy of signal integrity, circuit implementation and system level design.

Exploration of the signal integrity issues and common-mode impact on differential receiver circuits shows that common-mode backchannels with sub-50MHz bandwidth and swings as large as $100 \mathrm{mV}$ can be implemented without impact on the error rate of the forward link. The mostly digital implementation allows for flexibility in operation and robustness to the different sources of noise present in such a system. Measured results demonstrate the viability of using a common-mode backchannel for coefficient updates in a highspeed differential system.

\section{Acknowledgments}

The authors would like to acknowledge the help and support of the whole Rambus RaserX design team. A. Ho, V. Stojanović, and E. Alon also thank M. Ho, I. Stojanović, and M. Lee for their help and support.

\section{References}

[1] V. Stojanovic et. al "Adaptive Equalization and Data Recovery in a Dual-Mode (PAM2/4) Serial Link Transceiver," to be published. [2] J.T. Stonick et. al, "An adaptive PAM-4 5-Gb/s backplane transceiver in $0.25-/ \mathrm{spl} \mathrm{mu/m} \mathrm{CMOS,"} \mathrm{IEEE} \mathrm{Journal} \mathrm{of} \mathrm{Solid-State}$ Circuits, Mar. 2003

[3] T Gabara, "Phantom Mode Signaling in VLSI Systems," Conference on Advanced Research in VLSI, Mar. 2001

[4] J. Zerbe et al, "Equalization and Clock Recovery for a 2.5-10Gb/s 2-PAM/4-PAM Backplane Transceiver Cell," IEEE International Solid-State Circuits Conference, Feb. 2003, San Francisco. 